\title{
Niveles séricos de minerales en hembras de cría bovina en un establecimiento de la región de selva y pastizal pedemontano del norte de Salta.
}

\author{
Micheloud $\mathrm{JF}^{1,2^{*}}$, Suárez $\mathrm{VH}^{1}$, Martínez $\mathrm{GM}^{3}$, Rosa $\mathrm{DE}^{4}$, Ventura $\mathrm{MB}^{4}$, Mattioli $\mathrm{GA}^{4}$ \\ ${ }^{1}$ Grupo de Trabajo de Patología, Epidemiología e Investigación Diagnóstica. Área de Sanidad \\ Animal-IIACS Salta, Instituto Nacional del Tecnología Agropecuaria (INTA), Salta, Argentina \\ ${ }^{2}$ Facultad de Ciencias Agrarias y Veterinarias, Universidad Católica de Salta, Argentina \\ ${ }^{3}$ Grupo de Producción Animal EEA Salta, INTA \\ ${ }^{4}$ Laboratorio de Nutrición Mineral, Facultad de Ciencias Veterinarias, Universidad Nacional de \\ La Plata, Argentina
}

* Correspondencia: JF Micheloud. Grupo de Trabajo de Patología, Epidemiología e Investigación Diagnóstica. Área de Sanidad Animal-IIACS Salta/INTA. RN 68, km 72- Salta, Argentina.

E-mail: micheloud.juan@correo.inta.gob.ar

RESUMEN. Las deficiencias minerales son un problema de gran impacto productivo a nivel mundial. En el Noroeste argentino (NOA) la cría extensiva es una de las actividades económicas más importantes y está en franco crecimiento. En el NOA, los estudios sobre enfermedades carenciales son escasos y parciales. El presente trabajo tiene por objeto describir variaciones estacionales de los niveles séricos de calcio, fósforo, magnesio, cobre y zinc en vacas de cría y terneras de recría. En un establecimiento ganadero se seleccionaron 20 vacas y 20 terneras que fueron mantenidos en condiciones de pastoreo y sin suplementación externa. Se efectuaron muestreos de sangre a intervalos de 3 meses durante un año. Se determinaron niveles séricos de calcio, magnesio, cobre y zinc por espectrofotometría de absorción atómica y de fósforo por espectrofotometría uv-visible. Los resultados indican la existencia de hipocupremia moderada a leve en las terneras $(48,6 \pm 11 \mu \mathrm{g} / \mathrm{dL})$ durante todos los períodos muestreados. Las vacas presentaron valores promedio normales $(66 \pm 3,5 \mu \mathrm{g} / \mathrm{dL})$ pero cercanos al límite inferior durante algunos muestreos. Se observaron niveles subnormales de fósforo durante el verano (diciembre y marzo) en ambas categorías. Este trabajo sugiere que el cobre y el fósforo pueden comportarse como factores limitantes en las condiciones evaluadas.

SUMMARY. Serum levels of minerals in beef heifers and cows in the cloudforest of Salta, Argentina. Mineral deficiencies are a problem of great productive impact worldwide. In the Argentine Northwest (NOA) extensive breeding is one of the most important economic activities and it is in frank growth. In the NOA, studies on deficiency diseases are scarce and partial. The present work aims to describe seasonal variations of serum levels of calcium, phosphorus, magnesium, copper and zinc in beef cows and rearing calves. In a farm, 20 cows and 20 weaned heifer calves were selected, which were maintained under grazing conditions and without external supplementation. Blood samples were taken at intervals of 3 months for one year. Serum levels of calcium, magnesium, copper and zinc were determined by atomic absorption spectrophotometry and phosphorus by UV-visible spectrophotometry. The results indicate the existence of moderated hypocupremia in the calves $(48.6 \pm 11 \mu \mathrm{g} / \mathrm{dl})$ during all sampled periods. Cows presented normal values $(66 \pm 3.5 \mu \mathrm{g} / \mathrm{dl}$ ) but close to the lower limit during some sampling. Subnormal levels of phosphorus were observed during the summer (December and March) in both categories. This work confirms that copper and phosphorus can behave as limiting factors in the evaluated conditions.

Palabras clave: Deficiencia mineral, bovinos, calcemia, fosfatemia, magnesemia, cupremia

Key words: mineral deficiency, beef cattle, northwestern Argentina 


\section{Introducción}

En Salta la expansión ganadera es una realidad y ofrece una oportunidad importante para el desarrollo provincial (Rearte, 2011). La mayor parte de la actividad se realiza sobre pastizales naturales y pasturas perennes implantadas (gramíneas megatérmicas) (Brown et al., 2009). Estos sistemas (pastoriles) generalmente se asocian a carencias minerales, que afectan seriamente la productividad de los rodeos (McDowell 1985; Cunha, \& McDowell 2012). A pesar de esto, el NOA y en particular Salta tienen muy pocos antecedentes sobre el hallazgo o descripción de problemas sanitarios debidos a carencias minerales en el ganado.

El diagnóstico de carencias minerales en bovinos se realiza de rutina por evaluación de los niveles séricos en el rodeo (Underwood y Suttle, 2003). Estos niveles minerales se clasifican en rangos de normalidad, marginalidad o deficiencia, los cuales se pueden asociar a pérdidas productivas o problemas sanitarios (Mattioli, 2013).

El objetivo del presente trabajo fue evaluar los niveles séricos de calcio, fósforo, magnesio, cobre y zinc en hembras adultas y terneras a lo largo de un año en un establecimiento ganadero localizado en el área de selvas y pastizales pedemontanos del NOA.

\section{Materiales y Métodos}

Se evaluaron vacas de 1er parto y terneras de reposición de raza Brangus de un establecimiento agroganadero ubicado en la localidad de Coronel Cornejo, Departamento San Martin, al norte de la provincia de Salta (S 22ㅇ 76', W 63․84'). El establecimiento evaluado se encuentra ubicado en la ecoregión de selvas y pastizales pedemontanos de las Yungas, que representa el sistema de interfase entre las húmedas Selvas de Montaña (Yungas) y el bosque xerófilo (Brown \& Malizia 2004). Durante el periodo de estudio la alimentación fue a base de pasturas megatérmicas (Brachiaria brizantha var Mulato o Toledo y Panicum máximum var Tanzania).

El pastoreo era manejado en relación a la disponibilidad de forraje según la estación, donde en el caso de las vacas se usaban altas cargas en verano (13 vacas/ha) y moderadas en invierno, con escasa suplementación (maíz molido) a campo durante los periodos críticos. Por otro lado, las terneras pastorearon las mismas especies megatérmicas con suplemento de maíz molido, siendo la carga animal de 3 a 7 terneras por ha. El manejo reproductivo se basa en concentrar los partos en septiembre y octubre, destetando los precozmente terneros en enero y febrero. El agua de bebida estuvo constituida por fuentes naturales como arroyos y lagunas o de represas elaboradas para retener agua de lluvia o por bombeo de las fuentes antes citadas. Lamen- tablemente no existe información sobre las propiedades de estas fuentes que pudieran influir en el estatus mineral del ganado.

Se realizaron 4 muestreos de sangre (15-06-15; 15-09$15 ; 15-12-15$ y 15-03-16). Se seleccionaron del rodeo 20 vacas de 1 er parto y 20 terneras de destetadas precozmente en febrero. Vacas y terneras fueron identificados y mantenidos sin ningún tipo de suplementación mineral durante el ensayo. Las vacas al primer muestreo se hallaban en su $6^{\text {to }}$ mes de gestación, y en el de septiembre habían parido e iniciaban su lactancia; en diciembre el muestreo coincidió con el destete y al muestreo de marzo las vacas se encontra-ban al final del servicio.

Las muestras fueron centrifugadas para la obtención de suero libre de hemólisis y sobre este se realizaron las determinación de $\mathrm{Ca}, \mathrm{P}, \mathrm{Mg}$, Cu y Zn. Las concentraciones de $\mathrm{Ca}, \mathrm{Mg}$, $\mathrm{Cu}$ y $\mathrm{Zn}$ se midieron por espectrofotometría de absorción atómica de llama (Perkin Elmer AAnalyst 200), y las determinaciones de $P$ se realizaron mediante espectrofotometría UV-visible (Perkin ElmerLanbda25), según indicaciones del fabricante.

En cada fecha los niveles de $\mathrm{Ca}, \mathrm{P}, \mathrm{Mg}, \mathrm{Cu}$ y $\mathrm{Zn}$ se compararon entre categorías y fechas de muestreo usando una prueba de $t$, controlando previamente la presencia de heterocedasticidad por una prueba de bilateral de F. Se utilizó con tal fin el programa InfoStat.

\section{Resultados}

Los resultados obtenidos en vacas se presentan en al Tabla 1. En esta categoria, las diferencias entre períodos fueron significativas $(p<0,001)$ para el fósforo, descendiendo en los muestreos de diciembre y marzo.

Los resultados en las terneras se exponen en la Tabla 2. Esta categoria mostró valores de cobre significativamente $(p<0,05)$ más bajos que los de las vacas en todos los muestreos. Además todos los promedios estuvieron por debajo de las niveles considerados como fisiológicos (Underwood y Suttle, 2003). Con respecto al fósforo, al igual que en las vacas, las diferencias entre períodos fueron significativamente $(p<0,001)$ más bajas en los muestreos de diciembre y marzo.

\section{Discusión}

Este trabajo demuestra la existencia de niveles subnormales de $\mathrm{P}$ y $\mathrm{Cu}$ en ambas categorías (Rosa y Mattioli, 2002; Underwood and Suttle, 2003). El descenso en los niveles de $\mathrm{P}$ se observó en vacas luego del destete (diciembre) y al final del servicio en el servicio (marzo). Esto coincidiría con los mayores requerimientos de lac- 
tancia (NRC, 2000). Sin embargo, el mismo comportamiento se registró en las terneras de recría, las que mantuvieron altos requerimientos de crecimiento (NRC, 2000). Podría existir entonces un bajo aporte dietario del mineral durante la época lluviosa del verano. La carencia de $\mathrm{P}$ es fundamentalmente primaria y se debe a niveles bajos en las pasturas (Karn et al., 2001). Esta deficiencia ha sido muy estudiada en el Noreste Argentino (NEA), donde genera un gran impacto productivo (Mufarrege, 1999). Tras la deficiencia de sodio, la de $P$ es la carencia más común en los rumiantes alimentados en pastizales (Kinkaid, 1988). En el NOA los antecedentes son escasos o nulos. Según un informe INTAFAO (1981) se encontraron valores bajos de $P$ sérico aunque no evidencias clínicas de dicha carencia en animales de Salta y Tucumán.

Tabla 1: Niveles minerales en vacas (valor promedio \pm desvio estándar) en cada uno de los muestreos efectuados durante el ensayo. Las letras distintas indican diferencias significativas entre los distintos periodos.

\begin{tabular}{|c|c|c|c|c|c|}
\hline $\begin{array}{l}\text { Fecha de } \\
\text { muestreo }\end{array}$ & $\begin{array}{c}\mathrm{Ca} \\
(\mathrm{mg} / \mathrm{dL})\end{array}$ & $\begin{array}{c}P \\
(\mathrm{mg} / \mathrm{dL})\end{array}$ & $\begin{array}{c}\mathrm{Mg} \\
(\mathrm{mg} / \mathrm{dL})\end{array}$ & $\begin{array}{c}\mathrm{Cu} \\
(\mu \mathrm{g} / \mathrm{dL})\end{array}$ & $\begin{array}{c}\mathrm{Zn} \\
(\mu \mathrm{g} / \mathrm{dL})\end{array}$ \\
\hline Jun-15 & $\begin{array}{c}10,4 \\
\pm 1,3 \mathrm{a}\end{array}$ & $\begin{array}{r}5,2 \\
\pm 0,8 a\end{array}$ & $\begin{array}{r}2,8 \\
\pm 0,4 \text { a }\end{array}$ & $\begin{array}{c}69 \\
\pm 9 a\end{array}$ & $\begin{array}{r}139 \\
\pm 23 a\end{array}$ \\
\hline Sept-15 & $\begin{array}{r}9,5 \\
\pm 1,5 \mathrm{a}\end{array}$ & $\begin{array}{r}5,7 \\
\pm 0,7 \mathrm{a}\end{array}$ & $\begin{array}{r}2,3 \\
\pm 0,5 a\end{array}$ & $\begin{array}{c}63 \pm \\
12 a\end{array}$ & $\begin{array}{r}123 \\
\pm 30 a\end{array}$ \\
\hline Dic-15 & $\begin{array}{c}11,9 \\
\pm 1,9 \text { a }\end{array}$ & $\begin{aligned} & 3,7 \\
\pm & 0,3 \mathrm{~b}\end{aligned}$ & $\begin{array}{r}2,4 \\
\pm 0,4 a\end{array}$ & $\begin{array}{l}64 \pm \\
10 a\end{array}$ & $\begin{array}{c}105 \\
\pm 23 a\end{array}$ \\
\hline Mar-15 & $\begin{array}{c}11,1 \\
\pm 1,6 \mathrm{a}\end{array}$ & $\begin{aligned} & 3,7 \\
\pm & 0,4 \mathrm{~b}\end{aligned}$ & $\begin{array}{r}2,5 \\
\pm 0,3 a\end{array}$ & $\begin{array}{r}64 \\
\pm 10 a\end{array}$ & $\begin{aligned} & 126 \\
\pm & 20 a\end{aligned}$ \\
\hline
\end{tabular}

Tabla 2: Niveles minerales en terneras (valor promedio \pm desvió estándar) en cada uno de los muestreos efectuados durante e ensayo. Las letras distintas indican diferencias significativas entre los distintos periodos.

\begin{tabular}{|lcccccc|}
\hline $\begin{array}{l}\text { Fecha de } \\
\text { muestreo } \\
\text { (edad prom.) }\end{array}$ & $\begin{array}{c}\mathrm{Ca} \\
(\mathrm{mg} / \mathrm{dL})\end{array}$ & $\begin{array}{c}\mathrm{P} \\
(\mathrm{mg} / \mathrm{dL})\end{array}$ & $\begin{array}{c}\mathrm{Mg} \\
(\mathrm{mg} / \mathrm{dL})\end{array}$ & $\begin{array}{c}\mathrm{Cu} \\
(\mu g / \mathrm{dL})\end{array}$ & $\begin{array}{c}\mathrm{Zn} \\
(\mu g / \mathrm{dL})\end{array}$ \\
\hline $\begin{array}{l}\text { Jun-15 } \\
\text { (7 meses) }\end{array}$ & $\begin{array}{c}10,5 \\
\text { Sept-15 }\end{array}$ & 5,4 & 2,1 & 35 & 112 \\
\hline (10 meses) & $\pm 0,9$ & 6,4 & 2,3 & 55 & 121 \\
\hline Dic-15 & 11,1 & 3,1 & 2,2 & 42 & 116 \\
(13 meses) & $\pm 1,8 \mathrm{a}$ & $\pm 0,4 \mathrm{~b}$ & $\pm 0,3 \mathrm{a}$ & $\pm 14 \mathrm{~b}$ & $\pm 55 \mathrm{a}$ \\
\hline Mar-16 & 10,6 & 2,4 & 2,4 & 47 & 114 \\
(16 meses) & $\pm 1,8 \mathrm{a}$ & $\pm 0,3 \mathrm{~b}$ & $\pm 0,3 \mathrm{a}$ & $\pm 19 \mathrm{~b}$ & $\pm 22 \mathrm{a}$ \\
\hline
\end{tabular}

Los valores séricos de cobre deben ser superiores a 60 $\mu \mathrm{g} / \mathrm{dL}$ para ser considerados como normales; valores entre 59 y $30 \mu \mathrm{g} / \mathrm{dL}$ se consideran hipocupremias moderadas, y valores inferiores a $29 \mu \mathrm{g} / \mathrm{dL}$ como hipocupremias severas (Ramírez et al., 1998). Según los resultados observados las terneras presentaron deficiencia moderada durante todo el estudio. El rango de hipocupremia moderada es indicativo del agotamiento de la reserva hepática de Cu (Underwood y Suttle, 2003). Esto coincide con lo informado el hallazgo de bajos valores de cobre hepático en Salta (De Postiglione et al, 1984).

Si bien las vaquillonas presentaron niveles subnormales de Cu sérico durante todos los periodos muestreados el valor más bajo se registró post-destete $(7$ meses de edad) para luego elevarse en los muestreos sucesivos. Esta tendencia a la alza de los valores de la cupremia puede deberse al cambio de alimentación de los animales de leche a pasto. Normalmente la leche tiene bajos niveles de cobre (Underwood y Suttle, 2003). Durante la gestación, la madre, transfiere al hígado fetal una gran cantidad de $\mathrm{Cu}$, de modo que al nacer el ternero posee una concentración hepática mayor a la de su madre. Luego estos valores comienzan a descender debido al bajo aporte de la leche materna (Rosa \& Mattioli, 2002). Es esperable que los valores comiencen a recuperarse al comenzar la alimentación sólida.

Las vacas por su parte presentaron niveles normales pero cercanos al límite inferior, lo que sugiere un riesgo potencial. Las diferencias entre ambas categorías pueden ser atribuidas a que los animales en crecimiento activo tienen mayores demandas minerales y eso se expresa en los animales más jóvenes (Mattioli et al, 1996). La deficiencia de cobre puede ser secundaria a la presencia de antagonistas como molibdeno, hierro, etc. (Rosa \& Mattioli, 2002). Si bien los estudios sobre minerales en el NOA son escasos, hay antecedentes de déficit de cobre por exceso de molibdeno (Mo) y azufre (S) de finales de la década del 80 (Boggiato \& Ruksan, 1988, 1989). En establecimientos de cría de la provincia de Santiago del Estero se detectaron signos clínicos compatibles con deficiencia de cobre con una relación Cu:Mo desfavorable y con bajos niveles de $S$ en el agua (Boggiato \& Ruksan 1989). Un trabajo reciente donde se estudiaron 10 rodeos de cría en el departamento Anta en la provincia de Salta (Saravia et al., 2015) no se detectaron niveles que sugieran hipocuprosis.

La carencia de Mg es de gran importancia en la región centro del país, pero los niveles séricos obtenidos en este trabajo sugieren que este no fue un factor limitante para ninguna de las categorías bajo estudio. Según Cseh y Crenovich (1996) la hipomagenesemia, constituye la primera causa de muerte en vacas de cría en el sudeste de la provincia de Buenos Aires. Analíticamente, se considera que valores séricos de magnesio por debajo de $1,8 \mathrm{mg} / \mathrm{dl}$ constituyen factores de riesgo para la enfermedad (Suttle, 2010).

Otro elemento bajo estudio fue el $\mathrm{Zn}$. Este es un microelemento esencial para la salud del bovino; su carencia genera problemas sanitarios asociados a fallas inmunitarias, reproductivas, de crecimiento y de integridad de la piel y pezuñas (Rosa et al., 2008). En la bibliografía se considera que niveles séricos de $\mathrm{Zn}$ por debajo de $90 \mu \mathrm{g} / \mathrm{dl}$ son deficitarios (Suttle, 2010). En este trabajo tanto vacas como vaquillonas evidenciaron niveles normales para este micromineral. 
Este estudio muestra la presencia de niveles subnormales de $\mathrm{Cu}$ y P. Si bien por si mismos son indicativos de riesgo, serían necesarios más estudios para reconocer las causas y sus consecuencias productivas sobre las diferentes categorías bovinas y sistemas productivos y sobre estos datos diseñar planes de suplementación bien fundamentados.

\section{Agradecimientos}

Agradecimientos: a la Secretaría de Investigación de la Universidad Católica de Córdoba y a la Asociación

\section{Bibliografía}

Boggiato PR, Ruksan B. 1989. Molibdenosis en bovinos en el noroeste santiagueño. Rev. Arg. Prod. Anim. 9: 106.

Boggiatto PR y Ruksan B. 1988. Hipocuprosis secundaria de bovinos en Leales (Tucumán). Rev. Arg. Prod. Anim. 9: 9-10.

Brown AD, Blendinger PG, Lomáscolo T, García Bes P. 2009. Selva pedemontana de las Yungas: Historia natural, ecología y manejo de un ecosistema en peligro. Ediciones del subtrópico. Tucumán Argentina. 490 pp.

Brown AD, Malizia LR. 2004. Las selvas pedemontanas de las Yungas. Ciencia Hoy 14: 52-63.

Cseh SB y Crenovich H. 1996. Hipomagnesemia en el sudeste de la provincia de Buenos Aires. Archivos de Medicina Veterinaria. 28: 111-116.

Cunha TJ, McDowell LR. 2012. Nutrition of grazing ruminants in warm climates. Academic Press. New York. 443 pp.

De Postiglione MDGT, Bingley JB, Dwinger RH. 1984. Liver copper concentrations in catlle in Salta province, Argentina. Trop. Anim. Hlth Prod. 16: 188-189.

Informe Proyecto INTA-FAO n $(75 / 023)$. 1981. Sanidad Animal en el NOA: Informe Final. pp. 36-38.

Karn JF. 2001. Phosphorus nutrition of grazing cattle: a review. Anim. Feed Sci. Technol. 89: 133-153.

Kincaid R. 1988. Macroelementos para los ruminates. En: Church CD.El ruminate. Fisiología digestiva y nutrición. Ed. Acribia. Zaragoza España. pp. 373-390.

Mattioli GA. 2013. Nutrición Mineral y Vitamínica de Bovinos. CCB Academic Press. 2da Edición. La Plata, Argentina. 266 pp.

Mattioli GA, Ramirez CE, Giuliodori MJ, Tittarelli CM, Yano H, Matsui H. 1996. Characterization of cattle copper deficiency in the Magdalena District. Livest. Prod. Sci. 47:7-10.
McDowell LR, Kiatoko M, Lang CE, Fonseca HA, Vargas E, Loosli JK, Conrad JH. 1980. Latin American mineral research-Costa Rica. In: Verde LS and Fernandez A (Eds). Fourth World Conference on Animal Production, Buenos Aires, Argentina. pp 39-47.

Mufarrege DJ. 1999. Los minerales en la alimentación de vacunos para carne en la Argentina. Trabajo de divulgación técnica INTA, Mercedes, Corrientes. Argentina.

National Research Council (NRC) 2000. Nutrient Requirements of Beef Cattle. 7th ed. National Academy Press, Washington, DC (USA). 248 pp.

Ramírez CE, Mattioli GA, Tittarelli CM, Giuliodori MJ, Yano H. 1998. Cattle hypocuprosis in Argentina associated with periodically flooded soils. Livest. Prod. Sci. 55: 47-52

Rearte D. 2011. Situación actual y prospectiva de la ganadería argentina, un enfoque regional. Asociación Latinoamericana de Producción Animal 19: 46-49.

Rosa DE, Mattioli GA. 2002. Metabolismo y deficiencia de cobre en los bovinos. Analecta Vet. 22, 7-16.

Rosa DE, Fazzio LE, Picco SJ, Furnus CC, Mattioli GA. 2008. Metabolismo y deficiencia de zinc en bovinos. Analecta Vet. 28: 34-44.

Saravia FC, Martínez MV y Ávila GN. 2015. Relevamiento de la cupremia en rodeos de cría bovina del chaco semiárido salteño (Argentina). Rev. Vet. 26: 59-62.

Slutzky D. 2005. Los conflictos por la tierra en un área de expansión agropecuaria del NOA. La situación de los pequeños productores y los pueblos originarios. Rev. Interdiscipl. Est. Agr. 23: 59-10.

Suttle NF. 2010. The mineral nutrition of livestock. CABI Publishing. Wallingford, UK. 4th Edition. 587 pp.

Underwood EJ y Suttle NF. 2003. Los minerales en la Nutrición del ganado. Editorial Acribia. Zaragoza. España. 648 pp. 